\title{
Socioeconomic status and incidence of sudden cardiac arrest
}

\author{
Kyndaron Reinier PhD MPH, Elizabeth Thomas MSc, Douglas L. Andrusiek MSc, Tom P. Aufderheide MD, \\ Steven C. Brooks MD MHSc, Clifton W. Callaway MD PhD, Paul E. Pepe MD MPH, Thomas D. Rea MD MPH, \\ Robert H. Schmicker MS, Christian Vaillancourt MD MSc, Sumeet S. Chugh MD; and the Resuscitation \\ Outcomes Consortium Investigators
}

See related commentary by Huikuri on page 1692 and at www.cmaj.ca/lookup/doi/10.1503/cmaj.111245

\begin{abstract}
Background: Low socioeconomic status is associated with poor cardiovascular health. We evaluated the association between socioeconomic status and the incidence of sudden cardiac arrest, a condition that accounts for a substantial proportion of cardiovascularrelated deaths, in seven large North American urban populations.
\end{abstract}

Methods: Using a population-based registry, we collected data on out-of-hospital sudden cardiac arrests occurring at home or at a residential institution from Apr. 1, 2006, to Mar. 31,2007 . We limited the analysis to cardiac arrests in seven metropolitan areas in the United States (Dallas, Texas; Pittsburgh, Pennsylvania; Portland, Oregon; and Seattle-King County, Washington) and Canada (Ottawa and Toronto, Ontario; and Vancouver, British Columbia). Each incident was linked to a census tract; tracts were classified into quartiles of median household income.

Results: A total of 9235 sudden cardiac arrests were included in the analysis. For all sites combined, the incidence of sudden cardiac arrest in the lowest socioeconomic quartile was nearly double that in the highest quartile (incidence rate ratio [IRR] $1.9,95 \%$ confidence interval $[\mathrm{Cl}]$ 1.8-2.0). This disparity was greater among people less than 65 years old (IRR 2.7, $95 \% \mathrm{Cl} 2.5-3.0)$ than among those 65 or older (IRR 1.3, 95\% Cl 1.2-1.4). After adjustment for study site and for population age structure of each census tract, the disparity across socioeconomic quartiles for all ages combined was greater in the United States (IRR 2.0, 95\% Cl 1.9-2.2) than in Canada (IRR 1.8, 95\% Cl 1.62.0) ( $p<0.001$ for interaction).

Interpretation: The incidence of sudden cardiac arrest at home or at a residential institution was higher in poorer neighbourhoods of the US and Canadian sites studied, although the association was attenuated in Canada. The disparity across socioeconomic quartiles was greatest among people younger than 65 . The association between socioeconomic status and incidence of sudden cardiac arrest merits consideration in the development of strategies to improve survival from sudden cardiac arrest, and possibly to identify opportunities for prevention.
Competing interests: Tom Aufderheide is a consultant and advisory board member for JoLife, Medtronic, Take Heart America and Citizen CPR Foundation and is on the Volunteer Basic Life Support Subcommittee of the National American Heart Association. Clifton Callaway is a co-inventor of patents related to timing of defibrillation, licensed to Medtronic. No competing interests declared by Kyndaron Reinier,

Elizabeth Thomas, Douglas Andrusiek, Steven Brooks, Paul Pepe, Thomas Rea, Robert Schmicker, Christian Vaillancourt and Sumeet Chugh.

This article has been peer reviewed.

Correspondence to: Dr. Sumeet S. Chugh, sumeet.chugh@cshs.org

CMAJ 2011. DOI:10.1503 /cmaj.101512
A n estimated 250 000-300 000 sudden cardiac arrests occur each year in the United States, ${ }^{1}$ accounting for up to $63 \%$ of cardiac-related deaths annually. ${ }^{2}$ Despite advances in resuscitation, more than $95 \%$ of people who experience sudden cardiac arrest die, ${ }^{3}$ and up to $50 \%$ of sudden cardiac arrests occur in people who do not have a history of coronary artery disease. ${ }^{4}$

Socioeconomic status has been shown to predict many health outcomes, including all-cause mortality, ${ }^{5}$ prevalence of risk factors for cardiovascular disease ${ }^{6}$ and incidence of cardiovascular disease. $^{7-9}$ Despite this substantial literature, we found only three studies that examined the potential association between socioeconomic status and sudden cardiac arrest. Although the studies were small and conducted in single communities, each showed that the incidence of sudden cardiac arrest was significantly higher in lower socioeconomic areas. ${ }^{10-12}$ The Oregon Sudden Unexplained Death Study (Ore-SUDS) reported a 30\%-80\% higher incidence of sudden cardiac arrest in poorer neighbourhoods. A stronger association was observed among people less than 65 years old, a group for whom basic health care funding is not guaranteed in the United States. ${ }^{11}$

Low socioeconomic status may be linked to an increased risk of sudden cardiac arrest by a variety of mechanisms related to individual risk factors or health-promoting behaviours or neigh- 
bourhood characteristics. Individuals of lower socioeconomic status have been found to have a greater burden of risk factors for cardiovascular disease ${ }^{13}$ poorer control of established cardiovascular risk factors ${ }^{14}$ and longer delays in seeking hospital care for acute myocardial infarction. ${ }^{15}$ Numerous studies have also shown that disparities in health outcomes are apparent across the spectrum of socioeconomic status. ${ }^{16}$

A better understanding of community-level patterns in the distribution of sudden cardiac arrest may identify opportunities for improving survival, such as effective targeting of community training for cardiopulmonary resuscitation and placement of automated external defibrillators in lower-income communities. We tested the hypothesis that disparities in the incidence of sudden cardiac arrest by level of socioeconomic status would be evident in a variety of urban communities in the United States and Canada, and that this association would be most prominent among people less than 65 years old residing in US communities.

\section{Methods}

\section{Study sites and population}

The Resuscitation Outcomes Consortium is a clinical research network focusing on cardiopulmonary arrest and severe traumatic injury in 11 sites across the United States and Canada (see Appendix 1 at www.cmaj.ca/lookup/suppl/doi :10.1503/cmaj.101512/-/DC1). One of the registries it maintains is the ROC Epistry-Cardiac Arrest, an epidemiologic registry of out-ofhospital cardiac arrests assessed by emergency medical services personnel in each area. ${ }^{17}$

For our analysis, we included data from the registry for people who had a sudden cardiac arrest from Apr. 1, 2006, to Mar. 31, 2007. We focused our analysis on data from 7 of the 11 sites: 4 in the United States (Dallas, Texas; Pittsburgh, Pennsylvania; Portland, Oregon; and Seattle-King County, Washington) and 3 in Canada (Ottawa and Toronto, Ontario; and Vancouver, British Columbia). We excluded data from the remaining four sites because of insufficient or incomplete ascertainment of out-ofhospital cardiac arrests by census tract during the study period. Incomplete case reporting was observed from some participating agencies during finite periods. For these periods, cases and their covariates were imputed. ${ }^{3}$ Four of the seven sites included in our analysis had no missing incidence data; the remaining three sites had missing data for $2 \%$ to $8 \%$ of arrests.

Each US site included in this analysis from the ROC Epistry-Cardiac Arrest registry repre- sents a major metropolis and its immediate suburban and rural population. In contrast, the Canadian sites encompass large geographic areas, including smaller cities and large rural areas. Because the socioeconomic status of rural areas may be lower than that of urban areas, ${ }^{18}$ the inclusion of rural areas could have confounded our results. Therefore, for the Canadian sites, we restricted our analysis to the census metropolitan area of each site, which encompasses the city and its surrounding suburbs and rural areas, similar to the boundaries of the US sites. ${ }^{19}$

To restrict analysis to census tracts with complete information on out-of-hospital cardiac arrests, we used an automated algorithm to exclude tracts with incomplete coverage along the border of the service area of each study site, because service areas did not necessarily overlap with boundaries of census tracts. ${ }^{3}$

\section{Definition of sudden cardiac arrest}

The ROC Epistry-Cardiac Arrest registry contains data on out-of-hospital sudden cardiac arrests evaluated by emergency medical services personnel. The registry includes cardiac arrests for which resuscitation was attempted either by means of external defibrillation (by emergency personnel or lay responders) or by means of chest compressions performed by emergency medical services personnel. It also includes pulseless arrests for which resuscitation was not attempted for reasons including clear evidence confirming death or a do-not-attempt-resuscitation directive from a physician.

The cardiac arrests were classified as having an "obvious cause" (noncardiac) based on circumstances and evidence (e.g., chemical, traumatic or asphyxia-induced cardiac arrest) or "no obvious cause" if the cause was uncertain or evidence suggested a cardiac cause. ${ }^{17} \mathrm{We}$ restricted our analysis to out-of-hospital cardiac arrests with no obvious cause (likely primary sudden cardiac arrest) that occurred at home or in a residential institution such as an assisted-living facility or nursing home. We excluded arrests outside of the home because the socioeconomic status of the arrest location may not be a reliable indicator of individual or neighbourhood socioeconomic status for these people. Also, inclusion of people whose arrest occurred in a public place might confound estimates of incidence rates in metropolitan areas that typically have a large daily influx of people.

\section{Linking cardiac arrests to census tracts}

Census tracts, created by the US Census Bureau and Statistics Canada, are defined similarly as relatively small, stable areas with 1500 to 8000 people that are relatively homogenous with re- 
gard to population characteristics, economic status and living conditions. ${ }^{20,21}$

Geospatial measures from the location of the arrest were recorded for each out-of-hospital cardiac arrest. For the US sites, the census tract number was used. For the Canadian sites, the census tract number, or slightly truncated coordinates for latitude and longitude, or Universal Transverse Mercator coordinates were recorded. ArcGIS for Desktop (version 9.2, Esri, Redlands, California) was used to map the coordinates to a Canadian census tract.

\section{Socioeconomic indicators}

Individual- and family-level measures of socioeconomic status were not available in the ROC Epistry-Cardiac Arrest registry. For our analysis, we used the median household income within each census tract to characterize area-level socioeconomic status at the location of the arrest. We obtained these data from the US Census 2000 Summary File 3 and the 2006 Canadian census. We converted values to 2006 US dollars using the Consumer Price Index conversion factors to convert the US values (http://oregonstate.edu/cla/polisci/faculty -research/sahr/sahr.htm) and Bank of Canada average exchange rates to convert the Canadian values. On the basis of the median household incomes, census tracts in each study site were grouped into quartiles (low, low-medium, medium-high and high median household income).

\section{Statistical analysis}

To compare site-specific incidence across socioeconomic quartiles, we first conducted stratified analyses separately at each site to estimate overall incidence and incidence per quartile. For each site, the numerator for each quartile included all cardiac arrests at home or at residential institutions in census tracts in that quartile, and the denominator included the population of all census tracts in that quartile.

We used Poisson regression analysis to estimate incidence rate ratios (IRRs) and their 95\% confidence intervals (CIs) for each site. In these site-specific Poisson models, we evaluated the possible dose-response association between socioeconomic status and incidence of sudden cardiac arrest using models of rates per census tract, with numeric socioeconomic quartiles (from 1 to 4 ) to test for trend. This analysis was performed first for all ages combined, then stratified by age ( $\geq 65$ years and $<65$ years). Age 65 was chosen as the cut-off because it is the age at which Americans become eligible for Medicare, and thus patterns of health care use related to socioeconomic status may change at this age.

We also used Poisson regression analysis to estimate IRRs comparing the lowest to highest socioeconomic quartile pooled across all sites, as well as IRRs by country while adjusting for site. To test whether the linear trend across all quar-

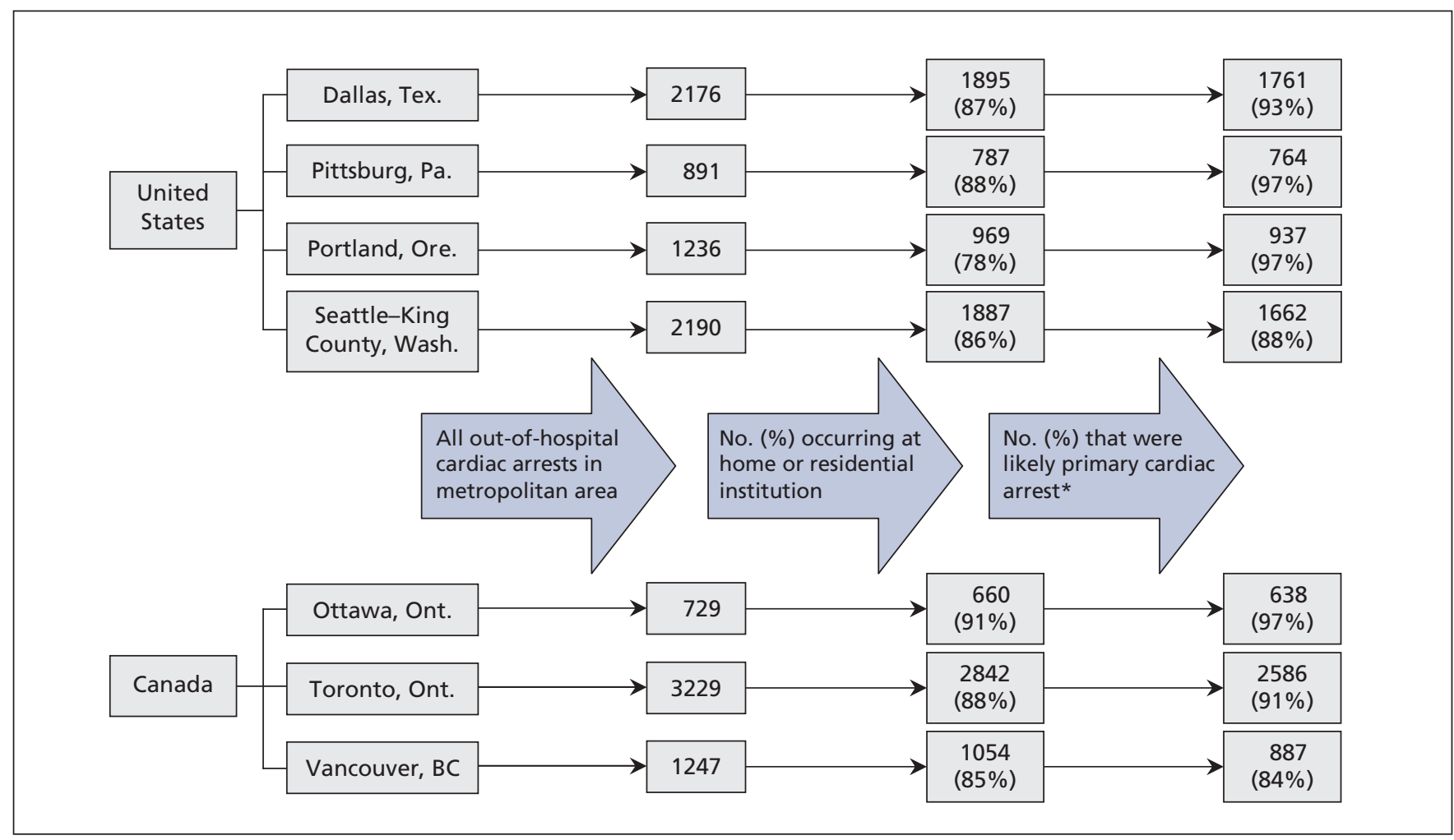

Figure 1: Selection of incidents of sudden cardiac arrest for analysis. *The arrest was not classified as having an obvious (noncardiac) cause. 
tiles differed in the United States and Canada, we used a single Poisson model adjusted for site with an interaction term for country and numeric socioeconomic quartile (from 1 to 4 ). Then, focusing only on the lowest and highest socioeconomic quartiles to assess the strength of the association between socioeconomic status and sudden cardiac arrest, we evaluated possible differences between the United States and Canada using a Poisson model with terms for site, categorical socioeconomic status (rather than numeric), and an interaction term for country by lowest socioeconomic quartile, with highest socioeconomic quartile as the reference. In these models, we adjusted for population age structure, with a term for the proportion of the population aged 65 or more in each census tract.

\section{Results}

A total of 11698 out-of-hospital cardiac arrests were identified in the seven study sites (Figure 1). Arrests occurring in public or private nonresidential locations were excluded (range 9\%-22\% across sites), as were arrests with an obvious noncardiac cause (range 3\%-16\% across sites). After these exclusions, $71 \%$ to $88 \%$ of the outof-hospital cardiac arrests in the study sites were retained for analysis, for a total of 9235 cardiac arrests likely of cardiac origin occurring in resi- dential locations ( $n=638$ to 2586 across sites).

The incidence of sudden cardiac arrest varied across the sites, with the US sites generally reporting a higher incidence than the Canadian sites (Table 1). The mean age at which sudden cardiac arrest occurred was lower in the US sites (range 64-69 years) than in the Canadian sites (range 69-70 years). The proportion of patients who were male ranged from $58 \%$ to $65 \%$ across sites (Table 1).

The median household income was similar across sites (range US\$50 000-US\$63 000), except in Pittsburgh, where it was substantially lower (Table 1). Income inequality, as measured by the ratio of highest to lowest quartile of median income, ranged from 2.2 to 3.2 across sites (Table 1).

\section{Site-specific comparisons}

At six of the seven sites, the incidence of sudden cardiac arrest was significantly higher in the lowest than in the highest socioeconomic quartile. Site-specific IRRs in the US sites ranged from 1.8 (Seattle-King County) to 2.2 (Dallas); in the Canadian sites, they ranged from 1.1 (Ottawa) to 2.3 (Toronto) (Table 2). The trend across quartiles was significant at each site, with lower socioeconomic status associated with higher incidence of sudden cardiac arrest $(p<0.001)$.

Table 1: Characteristics of out-of-hospital sudden cardiac arrests in the registry of the Resuscitation Outcomes Consortium in seven metropolitan areas in the United States and Canada, from Apr. 1, 2006, to Mar. 31, 2007

\begin{tabular}{|c|c|c|c|c|c|c|c|}
\hline \multirow[b]{2}{*}{ Characteristic } & \multicolumn{4}{|c|}{ United States } & \multicolumn{3}{|c|}{ Canada } \\
\hline & $\begin{array}{l}\text { Dallas, } \\
\text { Tex. }\end{array}$ & $\begin{array}{c}\text { Pittsburgh, } \\
\text { Pa. }\end{array}$ & $\begin{array}{l}\text { Portland, } \\
\text { Ore. }\end{array}$ & $\begin{array}{l}\text { Seattle-King } \\
\text { County, Wash. }\end{array}$ & $\begin{array}{l}\text { Ottawa, } \\
\text { Ont. }\end{array}$ & $\begin{array}{l}\text { Toronto, } \\
\text { Ont. }\end{array}$ & $\begin{array}{l}\text { Vancouver, } \\
\text { BC }\end{array}$ \\
\hline No. of sudden cardiac arrests* & 1761 & 764 & 937 & 1662 & 638 & 2586 & 887 \\
\hline Total populationt & 1995000 & 480000 & 1673000 & 1641000 & 1130000 & 3883000 & 2117000 \\
\hline $\begin{array}{l}\text { Annual incidence of sudden } \\
\text { cardiac arrest per } 100000 \neq\end{array}$ & 88.3 & 159.3 & 56.0 & 101.3 & 56.5 & 66.6 & 41.9 \\
\hline Age, yr, mean (SD) & $64(16)$ & $66(18)$ & $66(17)$ & $69(18)$ & $70(16)$ & $70(10)$ & $69(18)$ \\
\hline Age $\geq 65 \mathrm{yr}, \%$ & 53 & 53 & 56 & 59 & 63 & 67 & 62 \\
\hline Male sex, \% & 58 & 59 & 60 & 60 & 61 & 58 & 65 \\
\hline No. of census tracts included & 439 & 169 & 373 & 352 & 248 & 774 & 409 \\
\hline \multicolumn{8}{|l|}{ Median household income, \$§ } \\
\hline $\begin{array}{l}\text { Ratio of highest to lowest } \\
\text { quartile of median income }\end{array}$ & 3.2 & 2.5 & 2.2 & 2.4 & 2.5 & 2.5 & 2.2 \\
\hline \multicolumn{8}{|c|}{$\begin{array}{l}\text { Note: SD = standard deviation. } \\
\text { *Total number of arrests at home or at a residential institution that met criteria for analysis. } \\
\text { †Population of census tracts included in analysis, rounded to nearest thousand. } \\
\text { flncidence calculated for sudden cardiac arrests occurring at home or at a residential institution that met criteria for analysis; rates were not adjusted for age and sex. } \\
\text { §Values are shown in } 2006 \text { US dollars. }\end{array}$} \\
\hline
\end{tabular}


At all seven sites, disparities in the incidence of sudden cardiac arrest across socioeconomic quartiles were greatest among people less than 65 years old. Compared with the incidence in the highest quartile, the incidence in the lowest quartile was two- to fourfold greater in the US sites, and up to threefold greater in the Canadian sites (Table 2, Figure 2). As with all ages combined, disparities across socioeconomic quartiles among people younger than 65 were significant at all sites except Ottawa. Among people 65 years and older, the disparities were attenuated in

Table 2: Incidence rate ratios for out-ofhospital sudden cardiac arrest in lowest versus highest socioeconomic quartiles, by age

\begin{tabular}{|c|c|}
\hline Site; variable & $\begin{array}{l}\text { IRR in lowest v. } \\
\text { highest quartile } \\
(95 \% \mathrm{CI})\end{array}$ \\
\hline All US sites & $2.0 \quad(1.9-2.2)$ \\
\hline \multicolumn{2}{|l|}{ Dallas, Tex. } \\
\hline All ages & $2.2(1.9-2.6)$ \\
\hline Age $<65 \mathrm{yr}$ & $4.1 \quad(3.2-5.3)$ \\
\hline Age $\geq 65 \mathrm{yr}$ & $2.1 \quad(1.8-2.5)$ \\
\hline \multicolumn{2}{|l|}{ Pittsburgh, Pa. } \\
\hline All ages & $2.1 \quad(1.7-2.6)$ \\
\hline Age $<65 \mathrm{yr}$ & $2.7 \quad(1.9-3.7)$ \\
\hline Age $\geq 65 \mathrm{yr}$ & $1.6(1.2-2.1)$ \\
\hline \multicolumn{2}{|l|}{ Portland, Ore. } \\
\hline All ages & $2.1 \quad(1.8-2.6)$ \\
\hline Age $<65 \mathrm{yr}$ & $3.4 \quad(2.5-4.6)$ \\
\hline Age $\geq 65 \mathrm{yr}$ & $1.2(0.90-1.5)$ \\
\hline \multicolumn{2}{|c|}{ Seattle-King County, Wash. } \\
\hline All ages & $1.8(1.5-2.0)$ \\
\hline Age $<65$ yr & $2.4 \quad(1.9-3.0)$ \\
\hline Age $\geq 65 \mathrm{yr}$ & $1.2(0.98-1.4)$ \\
\hline All Canadian sites & $1.8(1.6-2.0)$ \\
\hline \multicolumn{2}{|l|}{ Ottawa, Ont. } \\
\hline All ages & $1.1 \quad(0.86-1.4)$ \\
\hline Age $<65 y r$ & $1.4 \quad(0.98-2.1)$ \\
\hline Age $\geq 65 \mathrm{yr}$ & $0.57(0.43-0.75)$ \\
\hline \multicolumn{2}{|l|}{ Toronto, Ont. } \\
\hline All ages & $2.3(2.0-2.6)$ \\
\hline Age $<65$ yr & $3.1 \quad(2.5-3.9)$ \\
\hline Age $\geq 65 \mathrm{yr}$ & $1.6(1.4-1.8)$ \\
\hline \multicolumn{2}{|l|}{ Vancouver, BC } \\
\hline All ages & $1.3(1.1-1.6)$ \\
\hline Age $<65$ yr & $1.7 \quad(1.2-2.3)$ \\
\hline Age $\geq 65 \mathrm{yr}$ & $0.84(0.64-1.1)$ \\
\hline All sites & $1.9(1.8-2.0)$ \\
\hline
\end{tabular}

the US and Toronto compared with those among younger individuals, and were reversed in Ottawa (Table 2, Figure 2).

\section{US-Canada comparisons}

For all sites combined, the incidence of sudden cardiac arrest in the lowest socioeconomic quartile was nearly double that in the highest quartile (Table 2, Figure 3). After adjusting for site and for population age structure of each census tract, we found that the disparity across socioeconomic quartiles was greater in the United States (IRR 2.0 v. 1.8) (Table 2), with a significant interaction term for country by lowest socioeconomic quartile
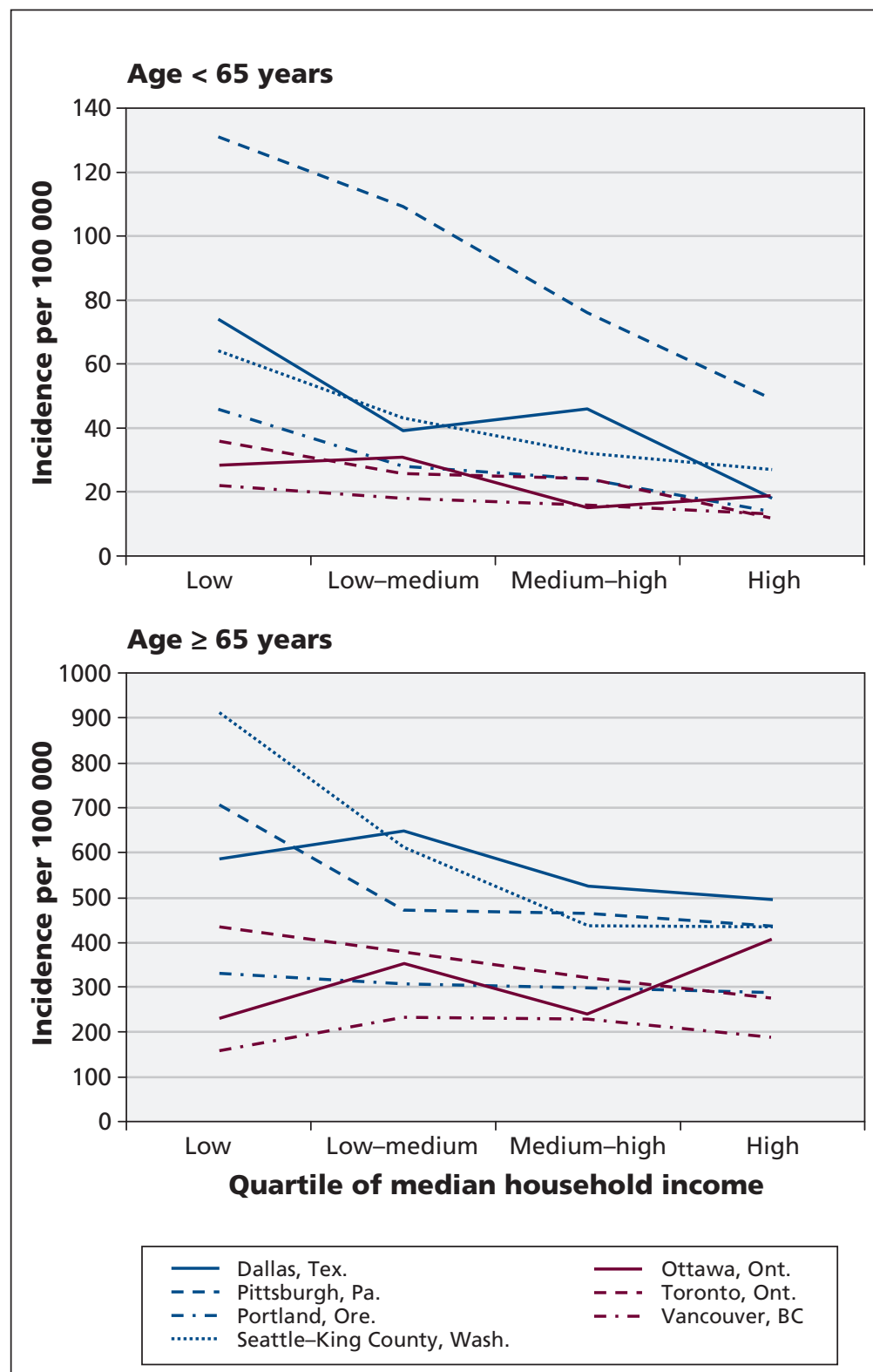

Figure 2: Incidence of sudden cardiac arrest in seven metropolitan areas in the United States and Canada among people less than 65 years old (top panel) and those 65 years and older (bottom panel) from Apr. 1, 2006, to Mar. 31, 2007, by quartile of median household income. 
$(p<0.001)$. In the model with socioeconomic status quartile modelled from 1 to 4 , the slope of the linear trend was significantly steeper in the United States than in Canada $(p<0.001)$ (Figure 3).

\section{Interpretation}

In this large, multicentre observational study, the incidence of sudden cardiac arrest was significantly higher in the neighbourhoods of lowest versus highest socioeconomic status in six of the seven metropolitan areas studied. We observed a significant linear trend of increasing incidence of cardiac arrest associated with decreasing quartile of median household income across all sites. The association between socioeconomic status and incidence of sudden cardiac arrest was most apparent among people less than 65 years old and was stronger in the United States than in Canada. Among people 65 years and older who experienced sudden cardiac arrest, significant disparities in incidence across socioeconomic quartiles were observed in only three of the seven sites.

Findings from our analysis are consistent with results from the Oregon Sudden Unexpected Death Study, which reported that the incidence of sudden cardiac arrest in the lowest socioeconomic quartile was about double that in the highest quartile, with a stronger association among patients less than 65 years old. ${ }^{11}$ Although the overall incidence of sudden cardiac arrest was higher among older patients, the disparities across socioeconomic quartiles that we observed among patients less than 65 translate to substantial socioeconomic disparities in premature death.

The persistent association between low socioeconomic status and poor health outcomes has

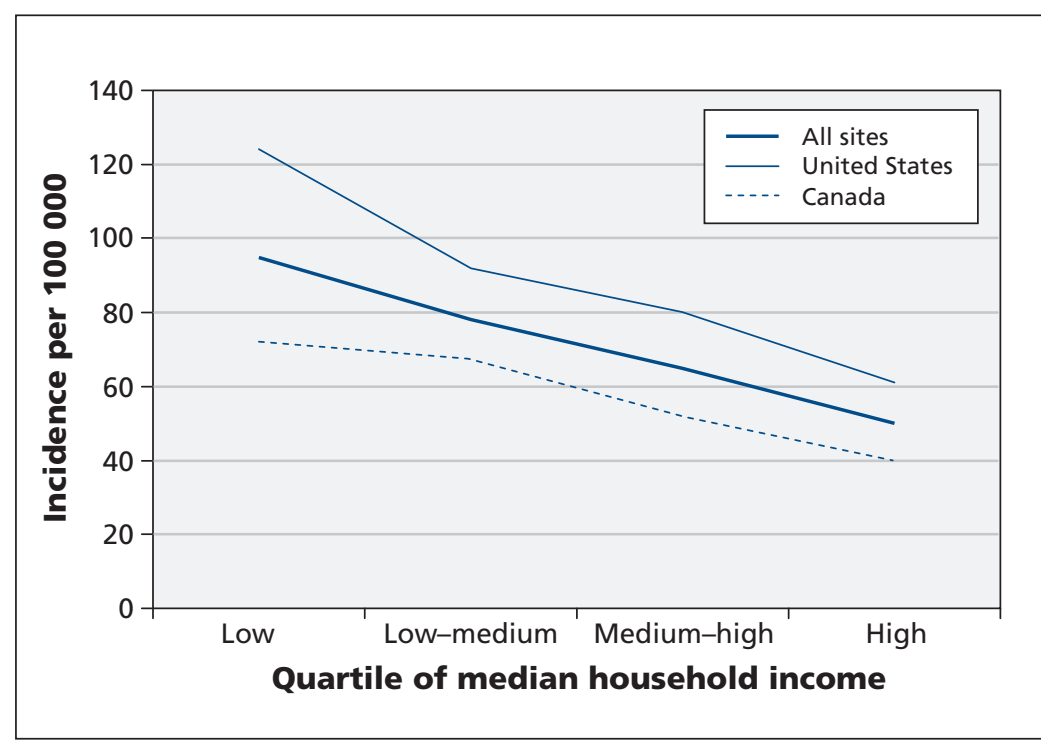

Figure 3: Incidence of sudden cardiac arrest among people of all ages in all sites combined from Apr. 1, 2006, to Mar. 31, 2007, by quartile of median household income. been observed in other studies, regardless of access to care. Despite universal health care in Canada, lower socioeconomic status has been associated with an increased burden of risk factors for cardiovascular disease, ${ }^{13}$ a decreased likelihood of cardiac catheterization following acute myocardial infarction ${ }^{22}$ and a decreased likelihood of receiving an implantable defibrillator among patients admitted to hospital because of heart failure. ${ }^{23}$ In the United States, among older individuals with Medicare, low socioeconomic status has been associated with late presentation with acute myocardial infarction. ${ }^{24}$

It is unclear why the disparity between socioeconomic status and incidence of sudden cardiac arrest in our study was somewhat more pronounced in the US sites combined than in the Canadian sites combined, although the difference was relatively small in magnitude, and the 95\% CIs overlapped. Income inequality was similar across US and Canadian sites. The population prevalence of established heart disease and history of myocardial infarction appear to be similar in the two countries. ${ }^{25,26}$ Measures of socioeconomic status at the census-tract level may reflect individual or neighbourhood socioeconomic status less accurately in Canada than in the United States; this may have resulted in an underestimation of true associations between socioeconomic status and sudden cardiac arrest in Canada. However, census tracts were similarly defined in each country, and median household income was computed in a similar manner.

Another possible explanation for the weaker association between socioeconomic status and incidence of sudden cardiac arrest in Canada may be the access to universal health care. In the United States, uninsured people less than 65 may have more undiagnosed cardiovascular disease or they may not seek care to manage risk factors for cardiovascular disease or to manage established heart disease. Such underdiagnosis or undertreatment could increase risk for sudden cardiac arrest. Our results showing a younger mean age of sudden cardiac arrest in the United States may be consistent with more poorly controlled cardiovascular disease. Previously published US data have shown socioeconomic disparities in the control of risk factors for cardiovascular disease and a decrease in differences after eligibility for Medicare at age $65 .{ }^{14}$

Patients of lower socioeconomic status in the United States have also been shown to delay seeking hospital care for acute myocardial infarction. ${ }^{15}$ A substantial proportion of patients who have sudden cardiac arrest have symptoms such as angina, dyspnea, nausea and syncope for minutes to hours before the arrest. ${ }^{27} \mathrm{~A}$ delay in 
calling for emergency medical services could result in a higher incidence of sudden cardiac arrest in the poorer, uninsured population.

Other data have shown Canadians to be more likely than their US counterparts to seek preventive care..$^{28}$ It is difficult from our current study and others to tease out the potential effects of health care systems, access to care and components of socioeconomic status on individual risk behaviour. Studies in other countries with nearuniversal access to health care have reported socioeconomic disparities in the incidence of sudden cardiac arrest in the United Kingdom ${ }^{12}$ and in cardiovascular-related mortality in Israel. ${ }^{29}$

Finally, we observed higher absolute rates of sudden cardiac arrest among people less than 65 years old in Pittsburgh than in the other sites. Although the registry data do not include health status information at the census tract level, data from the 2009 Behavioral Risk Factor Surveillance Study indicated that the burden of cardiovascular disease may be unusually high in Pittsburgh: the age-adjusted rate of death from coronary artery disease per 100000 was 153.5 in Allegheny County (Pittsburgh), compared with 141.6 in Tarrant County in Texas (near Dallas), 110.6 in Seattle-King County and 99.3 in Oregon..$^{30}$

\section{Strengths and limitations}

Among the strengths of our study are its large size, and the availability of data from seven diverse sites in the United States and Canada collected using consistent methodology. A standard definition of out-of-hospital sudden cardiac arrest was used across the study sites, as was an unbiased estimate of socioeconomic status based on high-quality census data. We restricted our analysis to sites with high levels of ascertainment of out-of-hospital cardiac arrests and to census tracts with likely complete reporting of arrests, which limited bias related to incomplete data.

Our study has several limitations. First, we may have introduced bias by including only arrests that occurred at home or at a residential institution. If people of lower socioeconomic status were more or less likely to have an arrest at home, this could affect the study's generalizability. However, because more than $80 \%$ of sudden cardiac arrests occur in a private residence, ${ }^{3}$ we were able to include most arrests.

Second, although the vast majority of sudden cardiac arrests (> 90\%) are due to a primary cardiac cause, ${ }^{31}$ a small minority of the arrests included in our study may have been due to noncardiac causes such as pulmonary embolism or aortic rupture.

Third, median household income at the census-tract level may be a more accurate mea- sure of socioeconomic status for younger than for older individuals. Some of the observed differences in the association between socioeconomic status and incidence of sudden cardiac arrest by age group may have been due to differences in how well socioeconomic status was measured for each age group. The use of only census-level measures of socioeconomic status is a potential limitation. Direct inference regarding the effects of individual- or neighbourhood-level socioeconomic status on individual risk of cardiac arrest may not be possible. However, measures of individual and census-tract socioeconomic status have produced similar estimates of association with various health outcomes in other studies. 32

\section{Conclusions}

The incidence of sudden cardiac arrest at home or at a residential institution was greater in lower- than in higher-income neighbourhoods in the US and Canadian sites studied, although the association was attenuated in Canada. The disparity across socioeconomic quartiles was greatest among people younger than 65. Populationbased interventions to reduce cardiovascular risk factors and treat unrecognized cardiovascular disease in lower-income communities may prove to be effective in reducing disparities in the incidence of sudden cardiac arrest. Also, effective targeting of training for cardiopulmonary resuscitation in lower-income communities with the highest incidence of sudden cardiac arrest may improve survival. Placement of automated external defibrillators in lower-income communities may also help, although only for arrests that occur outside the home.

\section{References}

1. Goldberger JJ, Cain ME, Hohnloser SH, et al. American Heart Association/American College of Cardiology Foundation/Heart Rhythm Society scientific statement on noninvasive risk stratification techniques for identifying patients at risk for sudden cardiac death. A scientific statement from the American Heart Association Council on Clinical Cardiology Committee on Electrocardiography and Arrhythmias and Council on Epidemiology and Prevention. J Am Coll Cardiol 2008;52:1179-99.

2. Zheng ZJ, Croft JB, Giles WH, et al. Sudden cardiac death in the United States, 1989 to 1998. Circulation 2001;104:2158-63.

3. Nichol G, Thomas E, Callaway CW, et al. Regional variation in out-of-hospital cardiac arrest incidence and outcome. JAMA 2008;300:1423-31.

4. Zipes DP, Wellens HJ. Sudden cardiac death. Circulation 1998; 98:2334-51.

5. Holme I, Helgeland A, Hjermann I, et al. Four-year mortality by some socioeconomic indicators: the Oslo study. J Epidemiol Community Health 1980;34:48-52.

6. Lee DS, Chiu M, Manuel DG, et al. Trends in risk factors for cardiovascular disease in Canada: temporal, socio-demographic and geographic factors. CMAJ 2009;181:E55-66.

7. Alboni P, Amadei A, Scarfò S, et al. In industrialized nations, a low socioeconomic status represents an independent predictor of mortality in patients with acute myocardial infarction. Ital Heart $J$ 2003;4:551-8.

8. Kaplan GA, Keil JE. Socioeconomic factors and cardiovascular disease: a review of the literature. Circulation 1993;88:1973-98. 
9. Loucks EB, Lynch JW, Pilote L, et al. Life-course socioeconomic position and incidence of coronary heart disease: the Framingham Offspring Study. Am J Epidemiol 2009;169:829-36.

10. Feero S, Hedges JR, Stevens P. Demographics of cardiac arrest: association with residence in a low-income area. Acad Emerg Med 1995;2:11-6.

11. Reinier K, Stecker EC, Vickers C, et al. Incidence of sudden cardiac arrest is higher in areas of low socioeconomic status: a prospective two year study in a large United States community. Resuscitation 2006;70:186-92.

12. Soo L, Huff N, Gray D, et al. Geographical distribution of cardiac arrest in Nottinghamshire. Resuscitation 2001;48:137-47.

13. Choinière R, Lafontaine $\mathrm{P}$, Edwards AC. Distribution of cardiovascular disease risk factors by socioeconomic status among Canadian adults. CMAJ 2000;162:S13-24.

14. McWilliams JM, Meara E, Zaslavsky AM, et al. Differences in control of cardiovascular disease and diabetes by race, ethnicity, and education: US trends from 1999 to 2006 and effects of medicare coverage. Ann Intern Med 2009;150:505-15.

15. Foraker RE, Rose KM, McGinn AP, et al. Neighborhood income, health insurance, and prehospital delay for myocardial infarction: the atherosclerosis risk in communities study. Arch Intern Med 2008;168:1874-9.

16. Adler NE, Ostrove JM. Socioeconomic status and health: What we know and what we don't. Ann N Y Acad Sci 1999;896:3-15.

17. Morrison LJ, Nichol G, Rea TD, et al. Rationale, development and implementation of the Resuscitation Outcomes Consortium Epistry-Cardiac Arrest. Resuscitation 2008;78:161-9.

18. Yost K, Perkins C, Cohen R, et al. Socioeconomic status and breast cancer incidence in California for different race/ethnic groups. Cancer Causes Control 2001;12:703-11.

19. Census dictionary, geographic units: census metropolitan area (CMA) and census agglomeration (CA). Ottawa (ON): Statistics Canada; 2001. p. 229.

20. US Census Bureau. Tracts and block numbering areas. Washington (DC): The Bureau; 2000.

21. Census dictionary, geographic units: census tract (CT). Ottawa (ON): Statistics Canada; 2001. p. 246.

22. Pilote L, Joseph L, Bélisle P, et al. Universal health insurance coverage does not eliminate inequities in access to cardiac procedures after acute myocardial infarction. Am Heart J 2003; 146:1030-7.

23. Udell JA, Juurlink DN, Kopp A, et al. Inequitable distribution of implantable cardioverter defibrillators in Ontario. Int J Technol Assess Health Care 2007;23:354-61.

24. Sheifer SE, Rathore SS, Gersh BJ, et al. Time to presentation with acute myocardial infarction in the elderly: associations with race, sex, and socioeconomic characteristics. Circulation 2000; 102:1651-6.

25. US Centers for Disease Control and Prevention (CDC). Prevalence of heart disease - United States, 2005. MMWR Morb Mortal Wkly Rep 2007;56:113-8.

26. Chow CM, Donovan L, Manuel D, et al. Regional variation in self-reported heart disease prevalence in Canada. Can J Cardiol 2005;21:1265-71.

27. Müller D, Agrawal R, Arntz HR. How sudden is sudden cardiac death? Circulation 2006;114:1146-50.

28. Katz SJ, Hofer TP, Manning WG. Physician use in Ontario and the United States: the impact of socioeconomic status and health status. Am J Public Health 1996;86:520-4.

29. Manor O, Eisenbach Z, Friedlander Y, et al. Educational differentials in mortality from cardiovascular disease among men and women: the Israel Longitudinal Mortality Study. Ann Epidemiol 2004; $14: 453-60$

30. US Centers for Disease Control and Prevention (CDC). Behavioral Risk Factor Surveillance Survey: chronic disease indicators. Atlanta (GA): CDC; 2009.

31. Hinkle LE Jr, Thaler HT. Clinical classification of cardiac deaths. Circulation 1982;65:457-64.

32. Krieger N. Overcoming the absence of socioeconomic data in medical records: validation and application of a census-based methodology. Am J Public Health 1992;82:703-10.

Affiliations: The Heart Institute (Reinier, Chugh), CedarsSinai Medical Center, Los Angeles, Calif.; the Department of Emergency Medicine (Reinier, Chugh), Oregon Health and Science University, Portland, Ore.; the Department of Biostatistics (Thomas, Schmicker), University of Washington Clinical Trial Center, Seattle, Wash.; British Columbia Ambulance Service and University of British Columbia (Andrusiek), Vancouver, BC; the Medical College of Wisconsin (Aufderheide), Milwaukee, Wis.; the Li Ka Shing Knowledge Institute at St. Michael's Hospital and the Program for Trauma, Emergency and Critical Care at Sunnybrook Health Sciences Centre (Brooks), Department of Medicine, University of Toronto, Toronto, Ont.; the University of Pittsburgh (Callaway), Pittsburgh, Pa.; the University of Texas Southwestern Medical Center (Pepe), Dallas, Tex.; Seattle-King County Public Health (Rea), Seattle, Wash.; and the Ottawa Hospital Research Institute (Vaillancourt), University of Ottawa, Ottawa, Ont. The complete list of Resuscitation Outcomes Consortium Investigators appears in Appendix 1 (www.cmaj.ca/lookup/suppl/doi:10.1503 /cmaj.101512/-/DC1).

Contributors: Douglas Andrusiek, Tom Aufderheide, Steven Brooks, Clifton Callaway, Paul Pepe, Thomas Rea, Christian Vaillancourt and Sumeet Chugh were involved in the acquisition of data. Elizabeth Thomas and Robert Schmicker performed data analysis. Kyndaron Reinier, Elizabeth Thomas, Douglas Andrusiek, Steven Brooks, Thomas Rea, Robert Schmicker and Sumeet Chugh interpreted the data. Kyndaron Reinier and Sumeet Chugh wrote the initial draft. All of the authors revised the manuscript for important intellectual content and approved the final version submitted for publication.

Funding: This study was supported by a cooperative agreement (grants 5U01 HL077863, HL077881, HL077871, HL077872, HL077866, HL077908, HL077867, HL077885, HL077887, HL077873 and HL077865) with the National Heart, Lung, and Blood Institute in partnership with the National Institute of Neurological Disorders and Stroke, the US Army Medical Research and Material Command, the Canadian Institutes of Health Research - Institute of Circulatory and Respiratory Health, Defence Research and Development Canada, the American Heart Association and the Heart and Stroke Foundation of Canada. Sumeet Chugh is the Pauline and Harold Price Professor of Electrophysiology at the Cedars-Sinai Heart Institute, Los Angeles, Calif. 